WSRC-TR-98-00125

Revision 0.0

Publication Date: March 1998

\title{
Characterization of DNAPL using Fluorescence Techniques
}

J. Rossabi

S. E. Nave

Westinghouse Savannah River Company

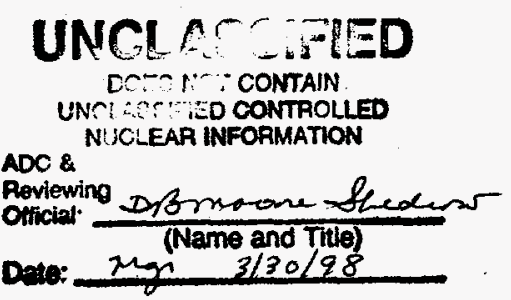

Savannah River Site

Aiken, SC, 29808

Prepared for the US Department of Energy under Contract DE-AC09-96SR18500

MASTER 


\section{DISCLAIMER}

This report was prepared as an account of work sponsored by an agency of the United States Government. Neither the United States Government nor any agency thereof, nor any of their employees, makes any warranty, express or implied, or assumes any legal liability or responsibility for the accuracy, completeness, or usefulness of any information, apparatus, product, or process disclosed, or represents that its use would not infringe privately owned rights. Reference herein to any specific commercial product, process, or service by trade name, trademark, manufacturer, or otherwise does not necessarily constitute or imply its endorsement, recommendation, or favoring by the United States Government or any agency thereof. The views and opinions of authors expressed herein do not necessarily state or reflect those of the United States Government or any agency thereof.

This report has been reproduced directly from the best available copy.

Available to DOE and DOE contractors from the Office of Scientific and Technical Information, P. O. Box 62, Oak Ridge, TN 37831; prices available from (423) 576-8401.

Available to the public from the National Technical Information Service, U. S. Department of Commerce, 5285 Port Royal Road, Springfield, VA 22161. 


\section{DISCLAIMER}

Portions of this document may be illegible electronic image products. Images are produced from the best available original document. 


\title{
Characterization of DNAPL using Fluorescence Techniques
}

\author{
J. Rossabi and S.E. Nave
}

Abstract

Dense non aqueous phase liquid (DNAPL) contaminants, comprised of chlorinated aliphatic compounds, are a major source of groundwater contamination at the Savannah River Site (SRS). To successfully remediate a site contaminated by DNAPLs, it is imperative that the slowly dissolving, non-aqueous phase source be found and removed. There are few technologies that can successfully and consistently detect DNAPLs in the subsurface either directly or by inferred measurements. Because of the use of chlorinated solvents to remove petroleum-based cutting oils and lubricants at SRS (and other manufacturing sites) in degreasing operations, waste solvents may contain small amounts of the oils and lubricants. This mixture will fluoresce when excited by light of wavelengths capable of being transmitted over optical fiber. Samples of DNAPL from the A/M area of SRS were analyzed to assess the possibilities of contaminant detection by fluorescence spectroscopy. The DNAPL sample exhibited a strong, distinct fluorescent spectrum when exposed to an appropriate excitation wavelength. A cone penetrometer-based, laser induced fluorescent system may be capable of providing direct detection of DNAPLs in the subsurface based on these results.

Introduction

Dense non aqueous phase liquid (DNAPL) contaminants are a major source of groundwater contamination at the Savannah River Site (SRS). These chlorinated organic contaminants are located in discrete pockets of the subsurface, both above and below the water table and continuously contaminate clean groundwater through dissolution (Looney et al., 1992). To successfully remediate a site contaminated by DNAPLs, it is imperative that the slowly dissolving, non-aqueous phase be found and removed. There are few technologies that can successfully and consistently detect DNAPLs in the subsurface either directly or by inferred measurements. Environmental scientists have had much success locating light non aqueous phase liquid (LNAPL) plumes at depth using cone penetrometer-based, fiber optic laser induced fluorescent techniques. The LNAPL compounds are normally petroleum-based, containing aromatic components, and will fluoresce when excited by wavelengths in the high ultraviolet to low visible range. These wavelengths can be transmitted through fiber optics over the distances required for many subsurface investigations (10 to 200 feet). DNAPLs are normally chlorinated aliphatic compounds that will only fluoresce when exposed to very low ultraviolet wavelengths. These low wavelengths cannot be transmitted through optical fiber as required by the cone penetrometer-based systems.

Fluorescent techniques have been used to locate petroleum by the oil industry for many years and have been suggested as a technique for locating DNAPL either directly or through the identification of fluorescent co-contaminants (Cohen and Mercer, pp 9-40, 1993), however, this method has not been widely applied to date. Some of the reasons for the modest use of fluorescence for DNAPL detection are:

- the broad fluorescent signal can rarely be used to positively identify a specific compound

- DNAPL is often located in thin, discrete, discontinuous ganglia rather than thick pools, so its easy to miss

- some natural soils fluoresce, making data interpretation difficult

- DNAPL is often inferred at a site rather than actually detected, so little is known about the composition and co-contaminants comprising the waste 
Dense chlorinated solvents were used in degreasing operations at SRS. As a result, the waste DNAPL solvents contain small amounts of the petroleum-based cutting oils and die lubricants. These oils tend to prefer to partition into non-polar solvents rather than water and can be transported in the DNAPLs through the subsurface. The lubricating and cutting oils (e.g., Fiske's 604 hot die lubricant) were comprised of a "cut" or mixture of petroleum distillates containing aliphatic, and aromatic compounds. The exact chemical make-up of these oils varied from batch to batch but proportions of compounds were fairly consistent. The lubricants also contained small amounts of other compounds such as graphite, organoclay, and aluminum.

DNAPL samples bailed from wells in the M area (MSB-3D and MSB-22) were not clear, as would be expected for pure PCE and TCE, but were a distinct amber color (Looney et al., 1992). This color may be due to a combination of natural organic matter with the solvents, however, the fraction of organic matter in the soils at the $M$ area is very low (foc $<0.0005$ from Looney et al., 1990) and the color of the DNAPL is different than the color of the sediments which tend to be red or tan. Another likely explanation for the amber colored solvents is the mixing of the solvents with cutting or lubricating oils. From conversations with personnel (meeting with James Weiderkehr, 9/11/96) who had operational experience at the time of the use of the chlorinated solvents, it is evident that the waste solvents were not clear before they were released to the outfalls and settling basins, probably due to mixing with the cutting oils and lubricants.

\section{Procedure}

We performed simple experiments on a bailed sample to determine the optical characteristics of the liquid DNAPL from well MSB-3D. Reagent grade PCE, ethyl alcohol, and dilutions of the DNAPL sample using the pure PCE as a diluent were also analyzed. For the absorption experiments, the samples were prepared in spectroscopic-grade quartz cuvettes to minimize attenuation losses in the ultraviolet wavelength range from the sample container and obtain a more accurate absorption profile. Standard borosilicate glass vials were used to contain the samples for the fluorescence measurements. Absorption spectra and fluorescent spectra were collected on the samples using fiber optic-based equipment. The results are given in the figures below.

Results

Figure 1 shows the absorption spectra of different concentration solutions of the DNAPL sample in a pure PCE diluent. Absorption or absorbance is defined through the Lambert-Beers equation as:

$$
A(a u)=\log _{10} \frac{I_{0}}{I}=a b C
$$

where $I_{\theta}$ is the initial intensity of a light source at a particular wavelength, $I$ is the intensity of the light (at the given wavelength) after it has passed through the analyte, $a$ is the absorptivity constant of the compound, and $b$ and $C$ are the path length of the light through the compound and the concentration of the absorbing species in grams per liter (Chen, 1987 and Silverstein et al., 1974). In figure 1, the numbers in the parentheses over the different DNAPL spectra identify the number of drops of bailed DNAPL added to $4 \mathrm{ml}$ of reagent grade PCE in a cuvette (the relative concentration of the PCE + DNAPL solution). Included in this figure are plots of pure PCE (no DNAPL added) and pure ethyl alcohol for reference. From these plots, it is clear that the peak absorption of pure PCE occurs between 250 and 280 $\mathrm{nm}$ with little absorption beyond $290 \mathrm{~nm}$. The peak absorption of the DNAPL, however, occurs at approximately $295 \mathrm{~nm}$ with significant absorption (> 0.1 au) still occurring as high as $390 \mathrm{~nm}$. These higher wavelengths are less attenuated by fiber optics so that more optical power can be delivered to the analyte. The more excitation power delivered, the higher the fluorescent response of the analyte and the better the detection limit of the system. 
Figure 1: SRS DNAPL Absorption

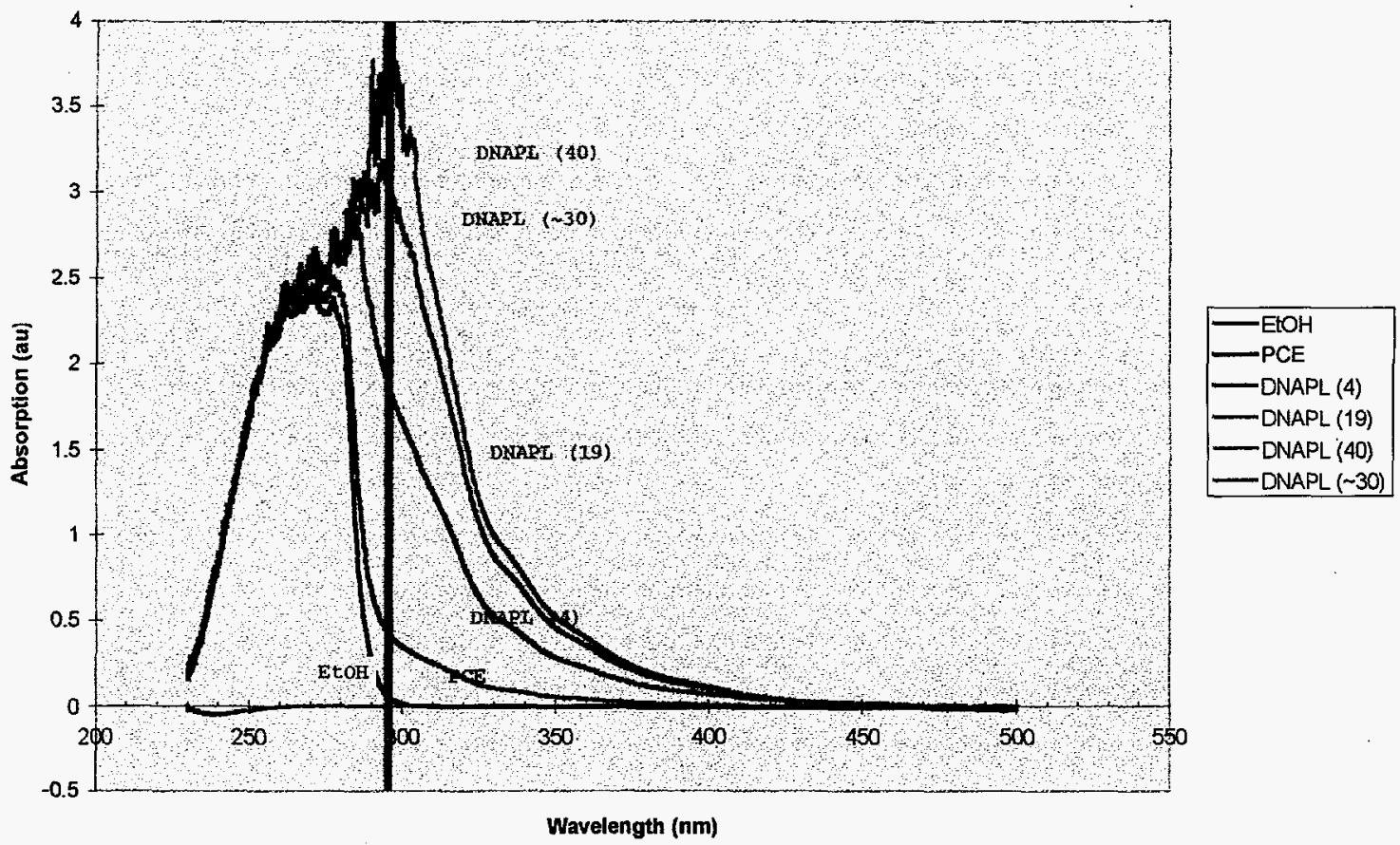

Figure 2 shows the fluorescence spectra of the DNAPL sample at various excitation wavelengths. The numbers in parentheses over the spectra indicate the excitation wavelength used. A mercury lamp with optical filters was used to select excitation lines. The spectra were all taken using a fiber optic delivery system focused on a $22 \mathrm{ml}$ vial containing the bailed DNAPL sample. Pure PCE, ethanol, and air in similar vials were also scanned for reference and comparison to the DNAPL spectra. The spectrometer was a Princeton Instruments CCD set for 1 second exposure and with slits at $50 \mu$. Low OH fibers with $600 \mu$ diameter cores were used to transmit the excitation and emission signals.

From the figure, it is clear that the DNAPL spectra at excitation wavelengths of both 365 and $405 \mathrm{~nm}$ have unique structure at approximately $440,480,520$, and $550 \mathrm{~nm}$. The fluorescent intensity of the two samples is also significantly higher than the spectra of the other samples. 
Figure 2: Fluorescence Spectra of DNAPL at SRS

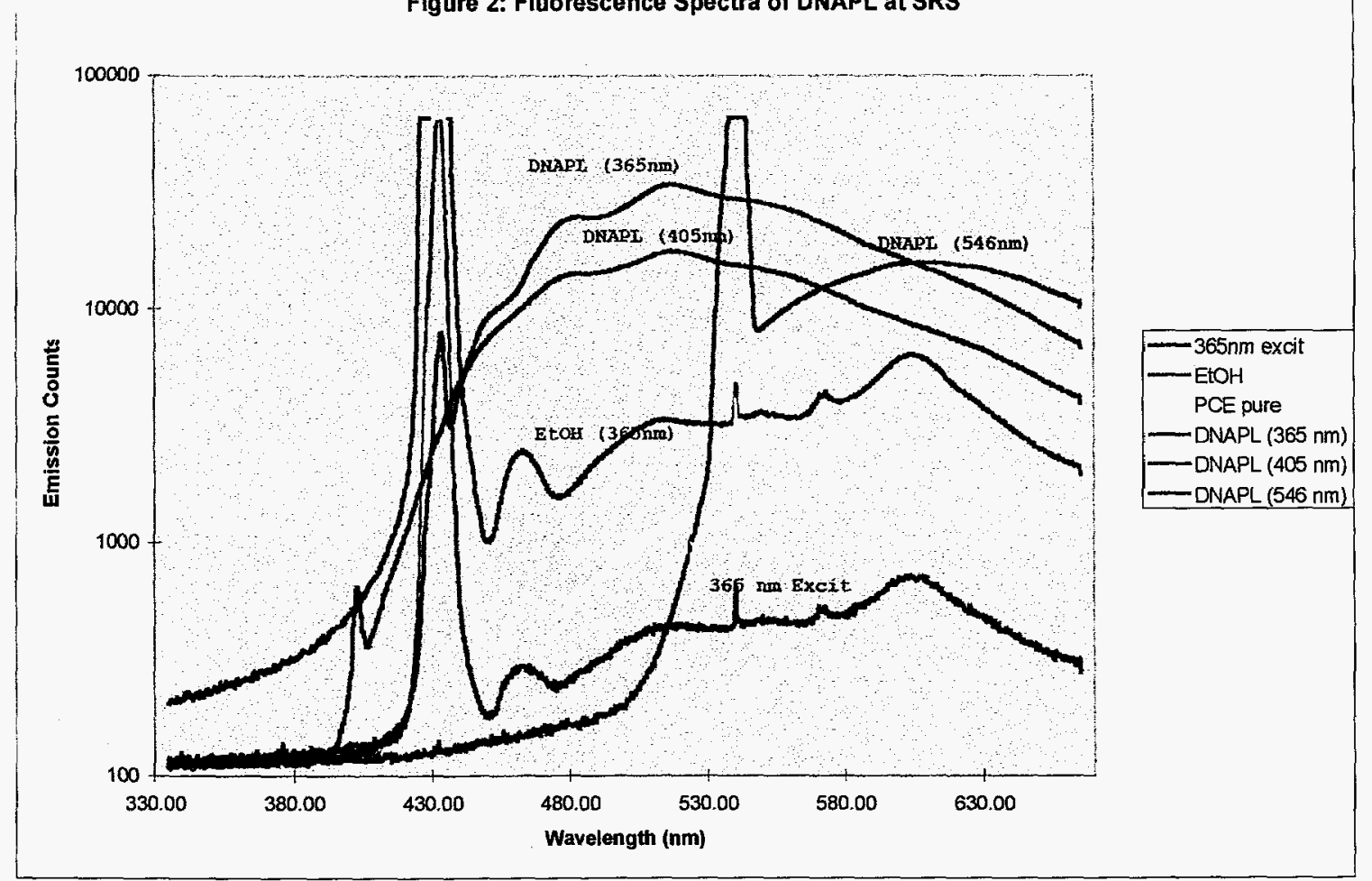

Figure 3 compares the background-corrected fluorescence spectra of the DNAPL samples collected using the mercury $(\mathrm{Hg})$ lamp and a low power, pulsed nitrogen laser with a principal emission line at $337 \mathrm{~nm}$. The spectral features at 480,520 , and 550 are discernible with the laser spectrum but not as clear as the higher-powered mercury lamp spectrum. These plots indicate that the source of excitation be selected for producing the maximum fluorescent signal from the contaminant. The proper source may not necessarily be the one with a wavelength closest to the maximum absorption wavelength. The amount of power capable of being transmitted through the optical fiber as well as the absorption efficiency of the light by the compound of interest must be considered. 
Figure 3: Comparison of DNAPL Fluorescence Spectra

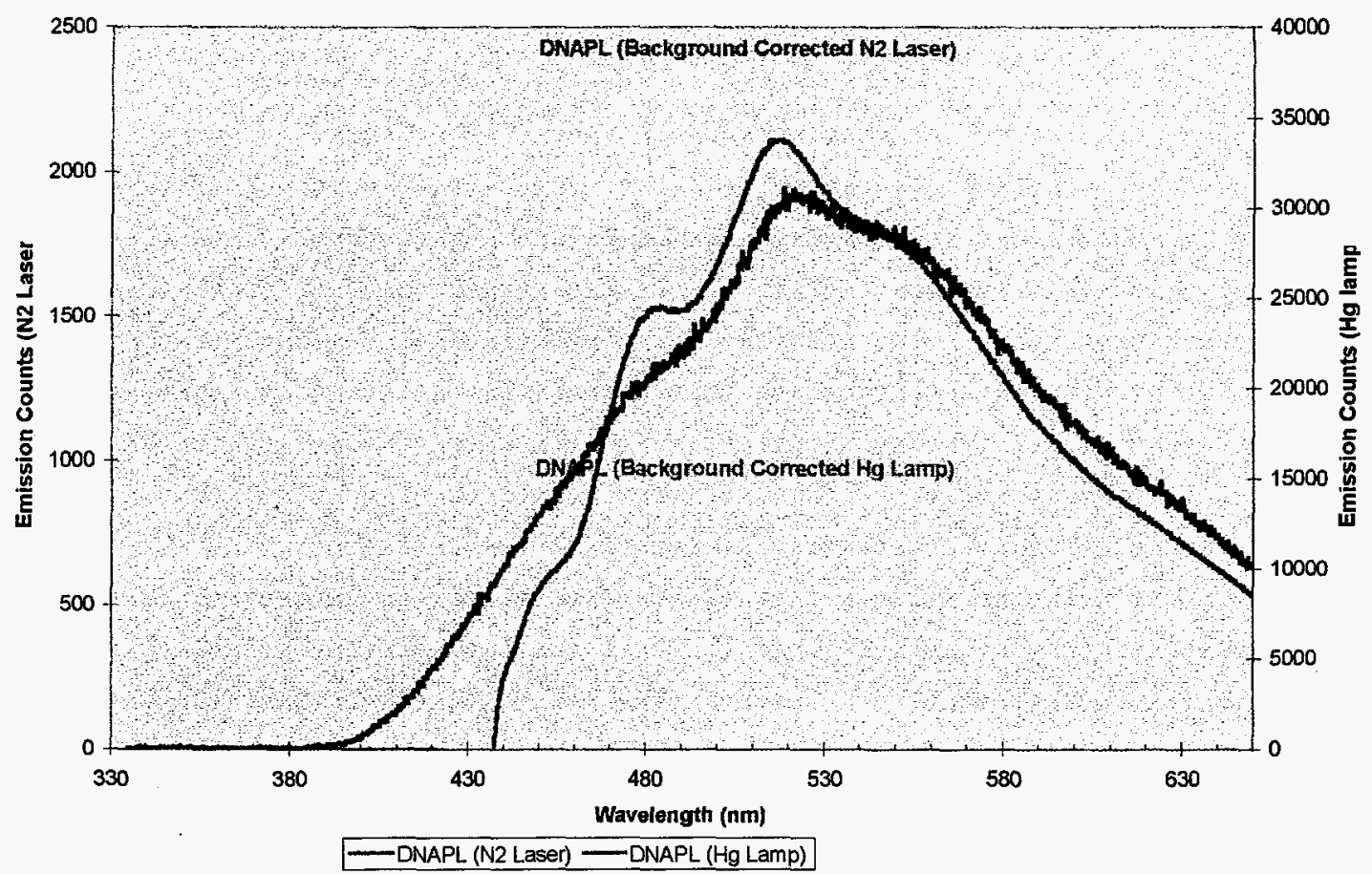

Conclusions

The relatively strong fluorescence response of the SRS sample at excitation wavelengths accessible to most fiber optic delivery systems shows the potential for using fluorescence as a DNAPL detection technique when the DNAPL has been co-disposed with compounds containing fluorophores. The same potential exists when chlorinated solvents have been released in an area containing leachable natural organic matter. In the latter case, the DNAPL is expected to extract and concentrate the natural organic matter, giving rise to significantly stronger fluorescent response (with respect to background) in areas where the DNAPL has accumulated. The data shown in these plots suggest that cone penetrometer-based laser induced fluorescence techniques may be applicable to DNAPL if the appropriate excitation wavelength is used and the proper emission wavelengths are detected. To aid in the selection of the optimal optical parameters for employing this method, samples of the oils and lubricants used in the metal fabricating processes and "degreased" by the chlorinated solvents should be obtained and analyzed for their optical characteristics.

\section{References}

Chen, H-L, 1987. Applications of laser absorption spectroscopy. In Radziemski, L.J., R.W. Solarz, and J.A. Paisner (Eds.), Laser spectroscopy and its applications (pp. 261-350). New York: Marcel Dekker, Inc.

Silverstein, R.M., G.C. Bassler, T.C. Morril, 1974. Spectrometric Identification of Organic Compounds, $3^{\text {rd }}$ ed. New York: John Wiley and Sons.

Looney, B.B., C.A. Eddy, M. Ramdeen, J. Pickett, V. Rogers, M.T. Scott, and P.A. Shirley, 1990. Geochemical and Physical properties of Soils and Shallow Sediments at the Savannah River Site, WSRCRP-90-1031, Westinghouse Savannah River Company, Savannah River Laboratory, Aiken, SC 29808. 
Looney, B.B., J. Rossabi, D.M. Tuck, J.E. Jordan, C.L. Bergren, R. Van Pelt, W.E. Jones, A. E. Stevenson, and B.S. Kristiansen, 1992. Assessing DNAPL Contamination, A/M-Area, SRS: Phase I Results, WSRC-RP-92-1302, Westinghouse Savannah River Company, Savannah River Laboratory, Aiken, SC 29808.

Cohen, R.M. and J.W. Mercer, 1993. DNAPL Site Evaluation. Boca Raton, FL: CRC Press, Inc. 